\title{
A projection operator approach to the Bose-Hubbard model
}

\author{
Anirban Dutta (1), C. Trefzger ${ }^{(2)}$, and K. Sengupta ${ }^{(1)}$ \\ (1) Theoretical Physics Department, Indian Association for the Cultivation of Science, Jadavpur, Kolkata-700032, India. \\ (2) ICFO - Institut de Ciencies Fotoniques, Mediterranean Technology Park, 08860 Castelldefels (Barcelona), Spain.
}

(Dated: June 4, 2018)

\begin{abstract}
We develop a projection operator formalism for studying both the zero temperature equilibrium phase diagram and the non-equilibrium dynamics of the Bose-Hubbard model. Our work, which constitutes an extension of Phys. Rev. Lett. 106, 095702 (2011), shows that the method provides an accurate description of the equilibrium zero temperature phase diagram of the Bose-Hubbard model for several lattices in two- and three-dimensions (2D and 3D). We show that the accuracy of this method increases with the coordination number $z_{0}$ of the lattice and reaches to within $0.5 \%$ of quantum Monte Carlo data for lattices with $z_{0}=6$. We compute the excitation spectra of the bosons using this method in the Mott and the superfluid phases and compare our results with mean-field theory. We also show that the same method may be used to analyze the non-equilibrium dynamics of the model both in the Mott phase and near the superfluid-insulator quantum critical point where the hopping amplitude $J$ and the on-site interaction $U$ satisfy $z_{0} J / U \ll 1$. In particular, we study the non-equilibrium dynamics of the model both subsequent to a sudden quench of the hopping amplitude $J$ and during a ramp from $J_{i}$ to $J_{f}$ characterized by a ramp time $\tau$ and exponent $\alpha$ : $J(t)=J_{i}+\left(J_{f}-J_{i}\right)(t / \tau)^{\alpha}$. We compute the wavefunction overlap $F$, the residual energy $Q$, the superfluid order parameter $\Delta(t)$, the equal-time order parameter correlation function $C(t)$, and the defect formation probability $P$ for the above-mentioned protocols and provide a comparison of our results to their mean-field counterparts. We find that $Q, F$, and $P$ do not exhibit the expected universal scaling. We explain this absence of universality and show that our results for linear ramps compare well with the recent experimental observations.
\end{abstract}

PACS numbers: 03.75.Lm, 05.30.Jp, 05.30.Rt

\section{INTRODUCTION}

Ultracold bosonic atoms in optical lattices provide us with an unique setup to study properties of bosons near a Mott insulator-superfluid (MI-SF) quantum critical point $\frac{1,2}{2}$. A careful analysis of such experimental bosonic systems in optical lattices show that their low-energy properties are well described by the BoseHubbard model $\underline{\underline{3}}$, which has already been theoretically studied using both analytical $\underline{\underline{4}}^{-\underline{\underline{6}}}$ and numerical ${ }^{\underline{\underline{7}}}$ techniques. The presence of such an experimental test bed has led to a plethora of new theoretical studies on the model ${ }^{-12}$. Many of the earlier analytical studies have concentrated on obtaining the phase diagram of the model by using mean-field theory $\underline{4,5}$, excitation energy computation ${ }^{6}$, and strong-coupling expansion for the boson Green function 12 . The results obtained by these methods have been compared to extensive quantum Monte Carlo (QMC) data 7,11 . Out of these methods, the strong-coupling expansion 12 (excitation energy computation ${ }^{-6}$ ) and the NPRG approach ${ }^{9}$ provide the closest match to QMC data in 2D (3D).

Recently, it has been realized that such ultracold bosonic systems also allow us easy access to the nonequilibrium dynamics of its constituent atoms near the MI-SF quantum critical point. The theoretical study of such quantum dynamics on various models has seen great progress in recent years $\frac{13}{3}$. Most of these works have either restricted themselves to the physics of integrable and/or one-dimensional (1D) models or concentrated on generic scaling behavior of physical observable for sudden or slow dynamics through a quantum critical point ${ }^{13}-17$. However quantum dynamics of specific experimentally realizable non-integrable models in higher spatial dimensions and strong coupling regime has not been studied extensively mainly due to the difficulty in handling quantum dynamics of plethora of states in the system's Hilbert space. The Bose-Hubbard model with onsite interaction strength $U$ and nearest neighbor hopping amplitude $J$, which provides an accurate description for ultracold bosons in an optical lattice, constitutes an example of such models. Most of the studies on dynamics of this model have concentrated on $d=1^{19}$, weak coupling regime ${ }^{20}$, and mean-field order parameter dynamics following a sudden ramp in the strong coupling regime ${ }^{21}-23$. Recent experiments ${ }^{2}$ clearly necessitate computation of dynamical evolution of several other quantities in higher dimensional Bose-Hubbard model in the strong-coupling regime $(U \gg J)$ beyond the mean-field theory and for arbitrary ramp time $\tau$. However, none of the works mentioned above presents an analysis of the non-equilibrium dynamics of the model beyond mean-field theory.

More recently, the authors of Ref. 24 have developed a theoretical formalism which enables one to analyze the dynamics of the Bose-Hubbard model beyond mean-field theory near the MI-SF critical point ${ }^{24}$. The method uses a projection operator technique which enables us to account for the quantum fluctuations over the meanfield theory perturbatively in $J_{f} / U(J(t) / U)$ and therefore yields accurate results as long $J_{f} / U(J(t) / U) \ll 1$ for sudden(ramp) dynamics. This allows one to treat sudden and slow ramps at equal footing near the MI- 
SF quantum critical point. As shown in Ref. 24, the projection operator method yields an accurate phase diagram and also provides an estimate of dynamically generated defect density which shows a qualitatively reasonable match with recent experimental results 2 . In the present work, we extend these results in several ways. First, we present a generic phase diagram of the BoseHubbard model as a function of the lattice coordination number $z_{0}$ and compare these results to the available QMC data for several one-, two-, and three-dimensional lattices. Our comparison demonstrates that the accuracy of the projection operator technique increases with $z_{0}$ reaching about $0.5 \%$ of the $\mathrm{QMC}$ data for lattices with $z_{0}=6$. Second, we compute the excitation spectrum using our approach and show that it yields the gapless Bogoliubov and gapped amplitude modes in the SF phase and the gapped particle and hole excitation modes in the MI phase. Third, we study the dynamics of the model for non-linear ramp of the hopping parameter $J$ from $J_{i}$ to $J_{f}$ characterized by a ramp time $\tau$ and exponent $\alpha$ : $J(t)=J_{i}+\left(J_{f}-J_{i}\right)(t / \tau)^{\alpha}$. We compute the fidelity susceptibility $F$, nearest-neighbor correlation between the bosons $B$, the defect formation probability $P$, and the residual energy $Q$ of the system following such a protocol and show that our result reproduce those of Ref. 24 for $\alpha=1$ as a special case. We also find the value of the optimal $\alpha$ which leads to minimal defect production for fast quenches (small $\tau$ ). Finally, we also compute the order parameter $\Delta(t)$, the order-parameter correlation function $C(t)$, the wavefunction overlap $F$, and the residual energy $Q$ subsequent to a sudden quench, discuss their properties, and provide explicit analytical expressions for $\Delta(t)$ and $Q$. We also provide a detailed comparison of the behavior of $\Delta(t)$ with that obtained from Gutzwiller mean-field theory.

The plan of the rest of the work is the following. In Sec. III we develop the projection operator formalism and apply it to obtain the equilibrium phase diagram of the Bose-Hubbard model for arbitrary $z_{0}$ and compute its excitation spectrum. This is followed by Sec. III, where we discuss the dynamics of the model both for sudden quench and non-adiabatic ramp of the hopping amplitude $J$. Finally we discuss our results and conclude in Sec. IV

\section{FORMALISM AND EQUILIBRIUM PHASE DIAGRAM}

In this section, we provide a detailed exposition of the projection operator formalism. In Sec. IIA we compute the MI-SF phase boundary using this formalism for various lattices while in Sec. IIB, we compute the low-energy excitation spectra of the MI and the SF phases.
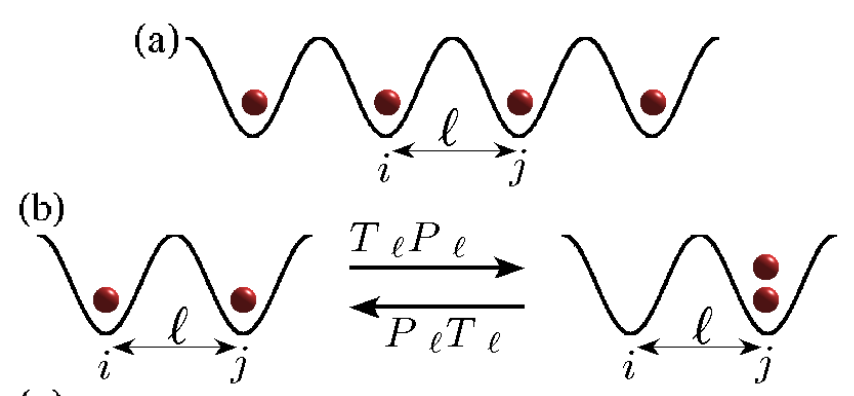

(c)

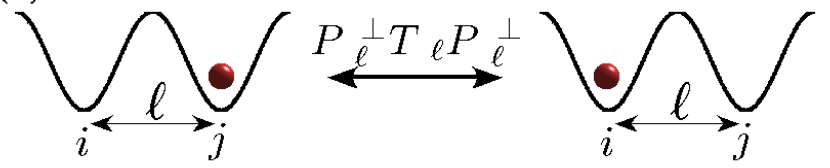

FIG. 1: (Color online) Schematic representation of the Mott state with $\bar{n}=1$. (b) Typical hopping process mediated via $T_{\ell}^{1}$. (c) Hopping process mediated via $T_{\ell}^{0}$. Notice that the states in (c) become part of the low-energy manifold near the critical point, while those in the right side of (b) do not and are always at an energy $U$ above the Mott state.

\section{A. Phase boundary}

The Hamiltonian of the Bose-Hubbard model is

$$
\begin{aligned}
\mathcal{H} & =T+H_{0}, \quad T=\sum_{\left\langle\mathbf{r r}^{\prime}\right\rangle}-J b_{\mathbf{r}}^{\dagger} b_{\mathbf{r}^{\prime}} \\
H_{0} & =\sum_{\mathbf{r}}\left[-\mu \hat{n}_{\mathbf{r}}+\frac{U}{2} \hat{n}_{\mathbf{r}}\left(\hat{n}_{\mathbf{r}}-1\right)\right]
\end{aligned}
$$

where $b_{\mathbf{r}}\left(\hat{n}_{\mathbf{r}}\right)$ is the boson annihilation (number) operator living on site $\mathbf{r}$ of a $d$-dimensional lattice with coordination number $z_{0}=\sum_{\left\langle\mathbf{r}^{\prime}\right\rangle_{\mathbf{r}}} 1$, and the chemical potential $\mu$ fixes the total number of particles. The exact solution of $\mathcal{H}$ is difficult even numerically due to the infinite dimensionality of the Hilbert space. A typical practice is to use the Gutzwiller ansatz $|\psi\rangle=\prod_{\mathbf{r}} \sum_{n} c_{n}^{(\mathbf{r})}|n\rangle$, and solve for $c_{n}^{(\mathbf{r})}$ keeping a finite number of states $n$ around the Mott occupation number $n=\bar{n}$. This yields the standard mean-field results with $c_{n}^{(\mathbf{r})}=c_{n}$ for homogeneous phases of the model ${ }^{10}$.

To build in fluctuations over such mean-field theory, we use a projection operator technique ${ }^{25}$. The key idea behind this approach is to introduce a projection operator

$$
P_{\ell}=|\bar{n}\rangle\left\langle\left.\bar{n}\right|_{\mathbf{r}} \times \mid \bar{n}\right\rangle\left\langle\left.\bar{n}\right|_{\mathbf{r}^{\prime}}\right.
$$

which lives on the link $\ell$ between the two neighboring sites $\mathbf{r}$ and $\mathbf{r}^{\prime}$ of the lattice. The hopping term $T$ can then be formally written as

$$
\begin{aligned}
T & =\sum_{\ell} T_{\ell}=\sum_{\ell}\left(T_{\ell}^{0}+T_{\ell}^{1}\right) \\
T_{\ell}^{0} & =P_{\ell}^{\perp} T_{\ell} P_{\ell}^{\perp}, \quad T_{\ell}^{1}=\left(P_{\ell} T_{\ell}+T_{\ell} P_{\ell}\right),
\end{aligned}
$$


where $P_{\ell}^{\perp}=\left(1-P_{\ell}\right)$. The advantage of the decomposition given by Eq. 3 is that it distinguishes between lowand high-energy tunneling processes as shown schematically in Fig. 1 for $\bar{n}=1$. The existence of a low-energy subspace for the model becomes more evident by rewriting the Bose-Hubbard Hamiltonian in the more convenient way $\mathcal{H}=\mathcal{H}_{0}+\mathcal{H}_{1}$, where

$$
\mathcal{H}_{0}=H_{0}+\sum_{\ell} T_{\ell}^{0}, \quad \mathcal{H}_{1}=\sum_{\ell} T_{\ell}^{1} .
$$

We can then define the low-energy subspace to be a set of states which are separated from the ground state of $\mathcal{H}_{0}$ by energies $O(J)$. These set of states can not be connected to each other by $\mathcal{H}_{1}$. For any two members, $\left|n_{1}\right\rangle$ and $\left|n_{2}\right\rangle$ of this set, one has $\left\langle n_{1}\left|\mathcal{H}_{1}\right| n_{2}\right\rangle=0$. In other words, $\mathcal{H}_{1}$ acting on any state $\left|n_{1}\right\rangle$ in these lowenergy subspace yields a state $\left|n_{1}^{\prime}\right\rangle$ which is necessarily separated from ground state of $\mathcal{H}_{0}$ by an energy $\mathrm{O}(U)$. Note that the states which are member of the low-energy subspace depend on the value of $J / U$. For example, the states schematically represented in panel (c) of Fig. 1 become members of the low-energy subspace near the MI-SF quantum critical point where $J \simeq J_{c}$; however, these states do not belong to the low-energy subspace for $J=0$.

In what follows, we shall use the projection operator technique to systematically chart out the effective lowenergy Hamiltonian by eliminating $\mathcal{H}_{1}$ from $\mathcal{H}$ to $\mathrm{O}(J)$. The canonical transformation operator $S$ which achieves this can be written as

$$
S \equiv S[J]=\sum_{\ell} i\left[P_{\ell}, T_{\ell}\right] / U .
$$

It can be easily checked that $\left[i S, H_{0}\right]=-\sum_{\ell} T_{\ell}^{1}$ so that the transformation eliminates $T_{\ell}^{1}[J]$ up to first order in $z_{0} J / U$. A standard expansion in $z_{0} J / U$ then leads to the effective Hamiltonian $H^{*}=\exp (i S) \mathcal{H} \exp (-i S)$ to
$\mathrm{O}\left(z_{0}^{2} J^{2} / U\right)$

$$
\begin{aligned}
H^{*} & =H_{0}+\sum_{\ell} P_{\ell}^{\perp} T_{\ell} P_{\ell}^{\perp}-\frac{1}{U} \sum_{\ell}\left[P_{\ell} T_{\ell}^{2}+T_{\ell}^{2} P_{\ell}\right. \\
& \left.-P_{\ell} T_{\ell}^{2} P_{\ell}-T_{\ell} P_{\ell} T_{\ell}\right]-\frac{1}{U} \sum_{\left\langle\ell \ell^{\prime}\right\rangle}\left[P_{\ell} T_{\ell} T_{\ell^{\prime}}-T_{\ell} P_{\ell} T_{\ell^{\prime}}\right. \\
& \left.+\frac{1}{2}\left(T_{\ell} P_{\ell} P_{\ell^{\prime}} T_{\ell^{\prime}}-P_{\ell} T_{\ell} T_{\ell^{\prime}} P_{\ell^{\prime}}\right)+\text { h.c. }\right]
\end{aligned}
$$

Note that the second order terms in $H^{*}$ involves effective hopping processes between adjacent links leading to spatial correlation between next-nearest neighbor sites; higher order terms in $z_{0} J / U$ systematically build such correlations between further neighbors. In this work, we restrict ourselves to $\mathrm{O}\left[\left(z_{0} J / U\right)^{2}\right]$.

Using $H^{*}$ one can now compute the variational ground state energy

$$
E=\langle\psi|\mathcal{H}| \psi\rangle=\left\langle\psi^{\prime}\left|H^{*}\right| \psi^{\prime}\right\rangle+\mathrm{O}\left(z_{0}^{3} J^{3} / U^{2}\right),
$$

where $\left|\psi^{\prime}\right\rangle=\exp (i S)|\psi\rangle$, and we use a Gutzwiller ansatz $\left|\psi^{\prime}\right\rangle=\prod_{\mathbf{r}} \sum_{n} f_{n}^{(\mathbf{r})}|n\rangle$, for the variational wavefunction $|\psi\rangle$ in the Mott limit $(S, J=0)$, where $|\psi\rangle=\left|\psi^{\prime}\right\rangle$. Note that $|\psi\rangle$ is not of the Gutzwiller form; it incorporates spatial correlation via $\exp (i S)$ factor. To obtain the variational energy $E$ in terms of the coefficients, we define the fields

$$
\begin{aligned}
\varphi_{\mathbf{r}} & =\left\langle\psi^{\prime}\left|b_{\mathbf{r}}\right| \psi^{\prime}\right\rangle=\sum_{n} \varphi_{\mathbf{r} n}=\sum_{n} \sqrt{n+1} f_{\mathrm{n}}^{*(\mathbf{r})} f_{\mathrm{n}+1}^{(\mathbf{r})} \\
\Phi_{\mathbf{r}} & =\left\langle\psi^{\prime}\left|b_{\mathbf{r}}^{2}\right| \psi^{\prime}\right\rangle=\sum_{n} \Phi_{\mathbf{r} n} \\
& =\sum_{n} \sqrt{(n+1)(n+2)} f_{\mathrm{n}}^{*(\mathbf{r})} f_{\mathrm{n}+2}^{(\mathbf{r})}
\end{aligned}
$$

Using the expressions of $\varphi_{\mathbf{r}}$ and $\Phi_{\mathbf{r}}$ in Eq. 8, one obtains, after some algebra, the expression for the variational energy $E \equiv E\left[\left\{f_{n}\right\} ; J\right]$ to be

$$
\begin{aligned}
E= & \sum_{\mathbf{r}, n}[-\mu n+U n(n-1) / 2]\left|f_{n}^{(\mathbf{r})}\right|^{2}-J \sum_{\left\langle\mathbf{r} \prime^{\prime}\right\rangle}\left\{\varphi_{\mathbf{r}}^{*} \varphi_{\mathbf{r}^{\prime}}-2 \Re \varphi_{\mathbf{r}, \bar{n}-1}^{*} \varphi_{\mathbf{r}^{\prime} \bar{n}}+J \bar{n}(\bar{n}+1) / U\left[\left|f_{\bar{n}}^{(\mathbf{r})}\right|^{2}\left|f_{\bar{n}}^{\left(\mathbf{r}^{\prime}\right)}\right|^{2}\right.\right. \\
& \left.\left.-\left|f_{\bar{n}+1}^{(\mathbf{r})}\right|^{2}\left|f_{\bar{n}-1}^{\left(\mathbf{r}^{\prime}\right)}\right|^{2}-f_{\bar{n}+1}^{*(\mathbf{r})} f_{\bar{n}-1}^{(\mathbf{r})} f_{\bar{n}-1}^{*\left(\mathbf{r}^{\prime}\right)} f_{\bar{n}+1}^{\left(\mathbf{r}^{\prime}\right)}\right]+2 J / U \Re \Phi_{\mathbf{r}, \bar{n}-2}^{*} \Phi_{\mathbf{r}^{\prime} \bar{n}}\right\}-J^{2} / U \sum_{\left\langle\mathbf{r r}^{\prime} \mathbf{r}^{\prime \prime}\right\rangle}\left\{2 \Re \left[\varphi_{\mathbf{r}, \bar{n}-1}^{*}(\bar{n}+1)\left|f_{\bar{n}}^{\left(\mathbf{r}^{\prime}\right)}\right|^{2}\right.\right. \\
& \left.+\varphi_{\mathbf{r} \bar{n}} \Phi_{\mathbf{r}^{\prime}, \bar{n}-2}^{*}-\varphi_{\mathbf{r} \bar{n}}^{*} \bar{n}\left|f_{\bar{n}-1}^{\left(\mathbf{r}^{\prime}\right)}\right|^{2}-\varphi_{\mathbf{r}, \bar{n}-1} \Phi_{\mathbf{r}^{\prime}, \bar{n}-1}^{*}\right] \varphi_{\mathbf{r}^{\prime \prime}}+2 \Re\left[\varphi_{\mathbf{r}, \bar{n}-1}^{*} \Phi_{\mathbf{r}^{\prime} \bar{n}}+\varphi_{\mathbf{r} \bar{n}} \bar{n}\left|f_{\bar{n}}^{\left(\mathbf{r}^{\prime}\right)}\right|^{2}-\varphi_{\mathbf{r}_{\bar{n}}}^{*} \Phi_{\mathbf{r}^{\prime}, \bar{n}-1}\right. \\
& \left.-\varphi_{\mathbf{r}, \bar{n}-1}(\bar{n}+1)\left|f_{\bar{n}+1}^{\left(\mathbf{r}^{\prime}\right)}\right|^{2}\right] \varphi_{\mathbf{r}^{\prime \prime}}^{*}+\varphi_{\mathbf{r} \bar{n}}^{*} \bar{n}\left[\left|f_{\bar{n}-1}^{\left(\mathbf{r}^{\prime}\right)}\right|^{2}-\left|f_{\bar{n}}^{\left(\mathbf{r}^{\prime}\right)}\right|^{2}\right] \varphi_{\mathbf{r}^{\prime \prime} \bar{n}}+\varphi_{\mathbf{r}, \bar{n}-1}(\bar{n}+1)\left[\left|f_{\bar{n}+1}^{\left(\mathbf{r}^{\prime}\right)}\right|^{2}-\left|f_{\bar{n}}^{\left(\mathbf{r}^{\prime}\right)}\right|^{2}\right] \varphi_{\mathbf{r}^{\prime \prime}, \bar{n}-1}^{*} \\
& \left.+2 \Re \varphi_{\mathbf{r} \bar{n}}^{*} \Phi_{\mathbf{r}^{\prime}, \bar{n}-1} \varphi_{\mathbf{r}^{\prime \prime}, \bar{n}-1}^{*}\right\},
\end{aligned}
$$

Note that the first three terms in the first line of Eq. 9 represent the mean-field energy functional, while the rest are corrections due to quantum fluctuations. Thus the projection operator method involves a systematic way of 
incorporating quantum fluctuations over the mean-field theory and we expect the results from this method to be accurate for larger $z_{0}$ where mean-field theory provides an accurate starting point.

The MI-SF phase diagram can be obtained by minimizing $E\left[\left\{f_{n}\right\} ; J\right]$ with respect to $\left\{f_{n}\right\}$ or by solving $i \hbar \partial_{t}\left|\psi^{\prime}\right\rangle=H^{*}[J]\left|\psi^{\prime}\right\rangle$ in imaginary time ${ }^{26}$. In this work, we are going to use the former technique and restrict ourselves to $\bar{n}=1$. Such phase diagrams for $2 \mathrm{D}$ and $3 \mathrm{D}$ square lattice are shown in Fig. 2(a) and Fig. 2(b) respectively. We note that the match with QMC data 11 is nearly perfect for 3D (Fig2(b)) where mean-field theory provides an accurate starting point. While in $3 \mathrm{D}$ the accuracy with QMC at the tip of the Mott lobe is $\sim 0.5 \%$, in $2 \mathrm{D}$ we find $J_{c} / U=0.055$ compared to the QMC value $0.061^{7}$ (red line in Fig. 2(a)). Here the match with QMC is not as accurate as in 3D; however it compares favorably to other analytical methods $\underline{\underline{6}}$.

To provide an accurate comparison of our method with other lattices, we note that the nature of the lattice affects $J_{c}$ only through $z_{0}$. We also note from Fig. 2(b) that the deviation of $J_{c}$ from QMC value is maximal at the tip of the Mott lobe. Thus to elucidate the $z_{0}$ and hence the lattice dependence of $J_{c}$ computed by the present method, we plot $J_{c}^{\text {tip }}$ (i.e., the value of $J_{c}$ at the tip of the Mott lobe) as a function of $z_{0}$ in Fig. 3. The comparison of corresponding QMC data for various lattices show that the method indeed becomes more accurate with increasing $z_{0}$.

Before ending this section, we would like to note that the inclusion of fluctuation in our method becomes apparent on computing the expectation $\left\langle T_{\ell}\right\rangle=$ $-J\langle\psi| b_{\mathbf{r}}^{\dagger} b_{\mathbf{r}^{\prime}}+$ h.c $|\psi\rangle$ in the MI phase. The mean-field theory provide a zero result for $\left\langle T_{\ell}\right\rangle$, while the projection operator method yields

$$
\begin{aligned}
\left\langle\psi\left|T_{\ell}\right| \psi\right\rangle= & \left\langle\psi^{\prime}\left|\exp (i S[J]) T_{\ell} \exp (-i S[J])\right| \psi^{\prime}\right\rangle \\
= & \left\langle\psi^{\prime}\left|T_{\ell}\right| \psi^{\prime}\right\rangle-\frac{1}{U}\left\langle\psi^{\prime}\right|\left[P_{\ell} T_{\ell}^{2}+T_{\ell}^{2} P_{\ell}-2 T_{\ell} P_{\ell} T_{\ell}\right] \\
& +\sum_{\left\langle\ell^{\prime}\right\rangle}\left\langle\psi^{\prime}\right|\left[P_{\ell} T_{\ell} T_{\ell^{\prime}}-T_{\ell} P_{\ell} T_{\ell}^{\prime}+\text { h.c. }\right]\left|\psi^{\prime}\right\rangle \quad(10)
\end{aligned}
$$

where we have kept terms up to $\mathrm{O}\left(J^{2} / U^{2}\right)$ and $\left\langle\ell^{\prime}\right\rangle$ denotes nearest neighbor links to $\ell$. In the MI phase, the first term of Eq. 10, which is also the mean-field result, vanishes, while the second fluctuation contribution from the remaining terms yields $\left\langle T_{l}\right\rangle=2 J^{2} \bar{n}(\bar{n}+1) / U$ in the homogeneous limit. We note that this agrees with fluctuation calculations of Ref. 6 .

\section{B. Excitation Spectrum}

To obtain the low-energy excitations, we consider a variational form for $\left|\psi^{\prime}\right\rangle$ which corresponds to perturba-
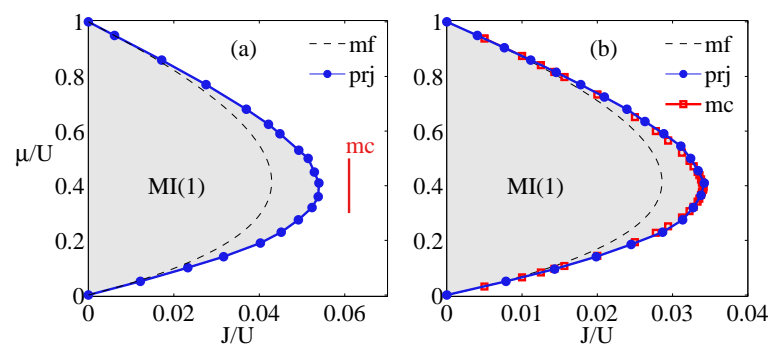

FIG. 2: (Color online) Phase diagram of the Bose-Hubbard model in 2D (a) and 3D (b). The blue dots and blue solid lines (black dashed line) indicate the phase diagram obtained by the projection operator (mean-field) method. The red squares indicate QMC data.

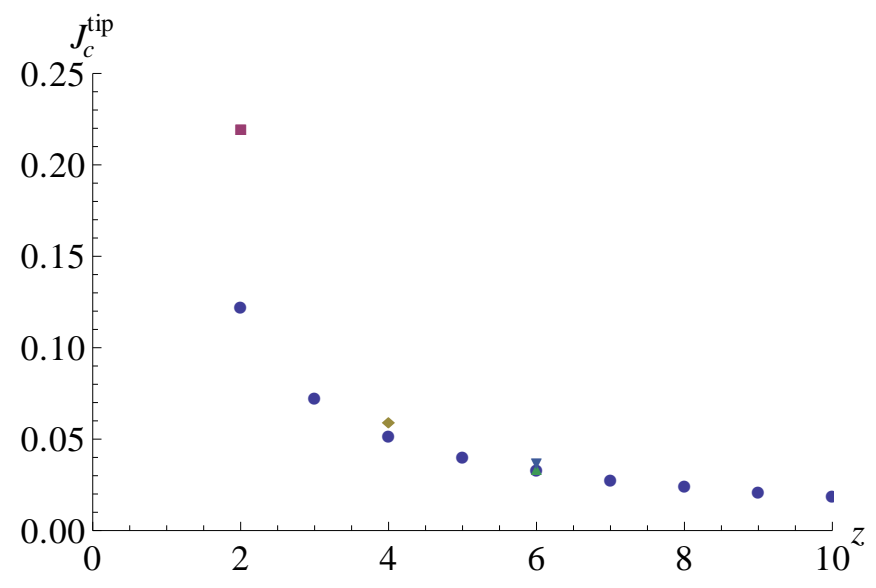

FIG. 3: (Color online) The plot of $J_{c}^{\text {tip }}$ as a function of $z_{0}$ as shown by blue circles. The red square, brown hexagon, green triangle, and the blue inverted triangle represents QMC data for $1 \mathrm{D}$ Bose Hubbard model $\left(z_{0}=2\right)$, 2D square lattice $\left(z_{0}=4\right), 3 \mathrm{D}$ cubic lattice $\left(z_{0}=6\right)$, and $2 \mathrm{D}$ triangular lattice $\left(z_{0}=6\right)$ respectively.

tion over the ground state value. This is given by

$$
\begin{aligned}
\left|\psi^{\prime}\right\rangle & =\prod_{\mathbf{r}} \sum_{n} f_{n}^{(\mathbf{r})}(t) \\
f_{n}^{(\mathbf{r})}(t) & =\left[f_{n}^{(0)}+\delta f_{n}^{(\mathbf{r})}(t)\right] e^{i \omega_{0} t}
\end{aligned}
$$

where $\delta f_{n}^{(\mathbf{r})}(t)$ represents small perturbation over the ground state value $f_{n}^{(0)}$ and can be expressed in momentum space as

$$
\delta f_{\mathrm{n}}^{(\mathbf{r})}(t)=u_{\mathbf{k}}^{n} e^{i(\mathbf{k} \cdot \mathbf{r}-\omega t)}+v_{\mathbf{k}}^{n} e^{-i(\mathbf{k} \cdot \mathbf{r}-\omega t)}
$$

Substituting Eqs. 11 and 12 in the Schrodinger equation $i \hbar \partial_{t}\left|\psi^{\prime}\right\rangle=H^{*}\left|\psi^{\prime}\right\rangle$, we obtain a set of equations for $u_{\mathbf{k}}^{n}$ and $v_{\mathbf{k}}^{n}$ which is given by

$$
\hbar \omega_{\mathbf{k}}\left(\begin{array}{c}
\vec{u}_{\mathbf{k}} \\
\vec{v}_{\mathbf{k}}
\end{array}\right)=\left(\begin{array}{cc}
A_{\mathbf{k}} & B_{\mathbf{k}} \\
-B_{\mathbf{k}} & -A_{\mathbf{k}}
\end{array}\right)\left(\begin{array}{c}
\vec{u}_{\mathbf{k}} \\
\vec{v}_{\mathbf{k}}
\end{array}\right) .
$$

Here the $\vec{u}_{\mathbf{k}}$ and $\vec{v}_{\mathbf{k}}$ are vectors with components $u_{\mathbf{k}}^{n}$ and $v_{\mathbf{k}}^{n}(n=0,1, \ldots)$, respectively and $A_{\mathbf{k}}$ and $B_{\mathbf{k}}$ are 
square matrices with elements $A_{\mathbf{k}}^{m n}$ and $B_{\mathbf{k}}^{n m}$. Since $0 \leq n \leq \infty$, in principle, Eq. 13 represents an infinitedimensional matrix equation; however, in the strong coupling regime where states with $n>3$ bosons are energetically costly, it is possible to truncate the arrays $\vec{u}_{\mathbf{k}}$ and $\vec{v}_{\mathbf{k}}$ by putting $u_{\mathbf{k}}^{n}, v_{\mathbf{k}}^{n}=0$ for $n>3$. In this case the column vector $\left(\vec{u}_{\mathbf{k}}, \vec{v}_{\mathbf{k}}\right)^{T}$ can be written as $\left(u_{\mathbf{k}}^{0}, u_{\mathbf{k}}^{1}, u_{\mathbf{k}}^{2}, u_{\mathbf{k}}^{3}, v_{\mathbf{k}}^{0}, v_{\mathbf{k}}^{1}, v_{\mathbf{k}}^{2}, v_{\mathbf{k}}^{3}\right)^{T}$. Thus the solution for the excitation spectrum reduces to the solution of a $8 \times 8$ matrix for each $\mathbf{k}$. In what follows we provide an analytical solution for $\omega_{\mathbf{k}}$ in the MI phase and a numerical plot of the excitation spectrum in the SF phase, where the algebra, for reasons mentioned below, turns out to be too complicated to allow a straightforward analytical result. We note here that our analysis amounts to generalization of the work in Ref. 27 which provides the excitation spectrum of the Bose-Hubbard model using mean-field theory.

In the MI phase, $f_{n}^{(0)}=\delta_{n 1}$ and $\hbar \omega_{0}$ can be shown to correspond to the ground state energy of the homogeneous MI state as obtained by putting $f_{1}^{(0)}=1$ in Eq. 9 . $\hbar \omega_{0}=-\mu-4 z_{0} J^{2} / U$. Further, one finds that in the MI phase the elements $A_{\mathbf{k}}^{n m} \sim \delta_{n m}$. Using the expression of $\omega_{0}$, these diagonal elements can be calculated to be

$$
\begin{aligned}
& A_{\mathbf{k}}^{00}=\mu-J z_{0}\left(1-2 x^{2}\right)-\frac{2 J^{2} z_{0}}{U}\left[\left(1-2 x^{2}\right)^{2} z_{0}-3\right] \\
& A_{\mathbf{k}}^{11}=-\frac{4 J^{2}\left(1-2 x^{2}\right) z_{0}}{U} \\
& A_{\mathbf{k}}^{22}=U-2 \mu-J z_{0}\left(1-2 x^{2}\right)+A_{\mathbf{k}}^{00} \\
& A_{\mathbf{k}}^{33}=\frac{4 J^{2} z_{0}}{U}+3 U-2 \mu .
\end{aligned}
$$

Note that in the limit $J=0$, these elements correspond to the on-site excitation energies of the different $|n\rangle$ states. The off-diagonal elements are given by

$$
\begin{aligned}
B_{\mathbf{k}}^{06} & =-\frac{3 \sqrt{2} J^{2} z_{0}\left(\left(1-2 x^{2}\right)^{2} z_{0}-1\right)}{U}=B_{\mathbf{k}}^{24} \\
B_{\mathbf{k}}^{15} & =-\frac{4 J^{2}\left(1-2 x^{2}\right) z_{0}}{U} .
\end{aligned}
$$

where $x=\sum_{a=1 . . d} \sin ^{2}\left(k_{a} / 2\right) / d$ and we have set the lattice spacing to unity. Diagonalization of the matrix in Eq. 13 leads to the excitation spectra given by

$$
\begin{aligned}
E_{\mathbf{k} 1}= & 3(U-\mu), \quad E_{\mathbf{k} 2}=\frac{\sqrt{\left(4 J^{2} z_{0}+U \mu\right)\left[U \mu-4 J^{2}\left(4 x^{2}-3\right)\right]}}{U}, \\
E_{\mathbf{k} 3}= & \frac{1}{2}\left[\left(\frac{-56 J^{4} z_{0}^{2}}{U^{2}}\left(\left(1-2 x^{2}\right)^{2} z_{0}-1\right)^{2}+\left(J^{2} z_{0}\left(\left(1-2 x^{2}\right)^{2} z_{0}+8\right)+12 J\left(1-2 x^{2}\right) z_{0} \mu+4 \mu^{2}\right)\right.\right. \\
& \left.\left.\frac{-8 J^{2} z_{0}}{U}\left(\left(1-2 x^{2}\right)^{2} z_{0}-1\right)\left(3 J\left(2 x^{2}-1\right) z_{0}-2 \mu\right)+2 U\left(3 J\left(2 x^{2}-1\right) z_{0}-2 \mu\right)+U^{2}\right)\right]^{1 / 2} \\
& +J z_{0}\left(2 x^{2}-1\right)+U-2 \mu
\end{aligned}
$$

Note that $E_{\mathbf{k} 2}$ corresponds to the hole branch while $E_{\mathbf{k} 3}$ corresponds to the particle branch. The energies of these branches differ from their mean-field counterparts in Ref. 27 via presence of additional fluctuation contribution which manifest then through $\mathrm{O}\left(J^{2} / U\right)$ terms. The plots of these excitation energies as a function of $x$ is shown in the left panel of Fig. 4 and matches qualitatively with its counterpart in Ref. 27.

A similar analysis for the superfluid phase can easily be carried out using the same algorithm described above. In this case, it turns out that the analytical expressions of $A_{\mathbf{k}}$ ( which now has off-diagonal terms) and $B_{\mathbf{k}}$ are prohibitively lengthy. We therefore resort to numerical solution of Eq.13 for several values of $x$. The result is shown in the right panel of Fig. 4. The qualitative features of the plots are again similar to the mean-field results of Ref. 27; however quantitative values of physical quantities such as the velocity of the Bogoliubov mode, $v_{g}$, differ. The difference with the mean-field result comes, again, from the presence of $\mathrm{O}\left(J^{2} / U\right)$ terms in the effective action and hence is small near the critical point.

\section{DYNAMICS}

We now demonstrate that the method elaborated in Sec. [II with minor modification allows one to address the dynamics of the Bose-Hubbard model. To this end, we are going to assume a protocol where the hopping amplitude $J \equiv J(t)$ changes in time from its initial value $J_{i}$ to some final value $J_{f}$. The necessary condition for our method to yield accurate result, as we shall demonstrate, is $z_{0} J(t) / U \ll 1$ at all times.

We begin with the Schrödinger equation for the time dependent Hamiltonian $\mathcal{H}[J(t)]$ which is given by

$$
i \hbar \partial_{t}|\psi\rangle=\mathcal{H}[J(t)]|\psi\rangle
$$

The solution of this equations is difficult due to the infinite dimensionality of the bosonic Hilbert space. How- 

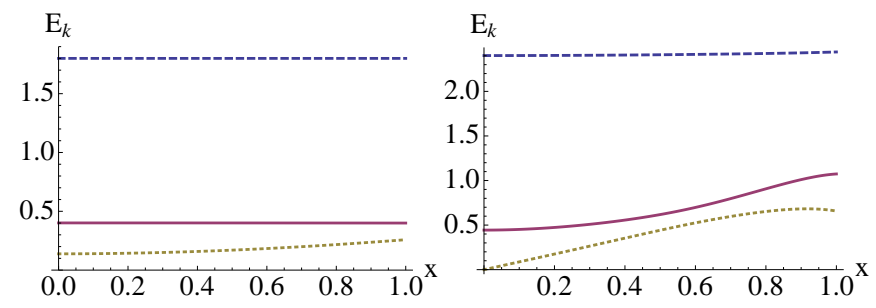

FIG. 4: (Color online) Left panel: Plot of the excitation spectra $E_{\mathbf{k} 1}$ (dashed blue line), $E_{\mathbf{k} 2}$ (solid purple line) and $E_{\mathbf{k} 3}$ (dotted yellow line) as a function of $x$ in the MI phase for $\mu=0.4 U$ and $J=0.15 J_{c}$. Right panel: Analogous plots for the excitation branches in the superfluid phase showing the gapless Bogoliubov mode and the gapped amplitude modes for $\mu=0.4 U$ and $J=1.3 J_{c}$.

ever, one notes that the contribution to the dynamics of the bosons, as long as $z_{0} J(t) / U \ll 1$, comes from a limited set of states which are members of the instantaneous low-energy subspace at any given instant $t$. To capture the contribution of the states in this low-energy subspace, we make a time-dependent transformation $\left|\psi^{\prime}\right\rangle=\exp (i S[J(t)])|\psi\rangle$, which eliminates $T_{\ell}^{0}[J(t)]$ up to first order from $\mathcal{H}[J(t)]$ at each instant, and leads to the effective Hamiltonian $H^{*}[J(t)]=$ $\exp (i S[J(t)]) \mathcal{H}[J(t)] \exp (-i S[J(t)]) . \quad$ This yields the equation

$$
\left(i \hbar \partial_{t}+\partial S / \partial t\right)\left|\psi^{\prime}\right\rangle=H^{*}[J(t)]\left|\psi^{\prime}\right\rangle .
$$

We note that the additional term $\partial S / \partial t$ takes into account the possibility of creation of excitations during the time evolution with a finite ramp rate $\tau^{-1}$. The above equation yields an accurate description of the ramp with $H^{*}[J(t)]$ given by Eq. 6 for $J(t) / U \ll 1$. Note that this does not impose a constraint on magnitude of $\tau$; it only restricts $J_{f} / U$ and $J_{i} / U$ to be small. Thus the method can treat both "slow" and "fast" ramps at equal footing. Substituting $\left|\psi^{\prime}\right\rangle=\prod_{\mathbf{r}} \sum f_{n}^{(\mathbf{r})}|n\rangle$, we obtain a set of coupled equations for the coefficients $\left\{f_{n}\right\}$

$$
\begin{gathered}
i \hbar \frac{\partial f_{\mathrm{n}}^{(\mathbf{r})}}{\partial t}=\frac{i \hbar}{U} \frac{\partial J}{\partial t}\left[\sqrt{n} f_{\mathrm{n}-1}^{(\mathbf{r})} \overline{\partial \alpha}_{\mathbf{r}}+\sqrt{n+1} f_{\mathrm{n}+1}^{(\mathbf{r})} \bar{\partial}_{\mathbf{r}}{ }^{*}\right] \\
+\chi_{\mathrm{n}}^{(\mathbf{r})} f_{\mathrm{n}}^{(\mathbf{r})}-J\left[\sqrt{n} f_{\mathrm{n}-1}^{(\mathbf{r})} \bar{\alpha}_{\mathbf{r}}+\sqrt{n+1} f_{\mathrm{n}+1}^{(\mathbf{r})} \bar{\beta}_{\mathbf{r}}^{*}\right] \\
-\frac{J^{2}}{U}\left[\sqrt{n(n-1)} f_{\mathrm{n}-2}^{(\mathbf{r})} \bar{\eta}_{\mathbf{r}}+\sqrt{(n+1)(n+2)} f_{\mathrm{n}+2}^{(\mathbf{r})} \bar{\xi}_{\mathbf{r}}^{*}\right]
\end{gathered}
$$

where the fields $\chi_{\mathbf{n}}^{(\mathbf{r})}, \bar{\alpha}_{\mathbf{r}}, \bar{\beta}_{\mathbf{r}}{ }^{*}, \bar{\eta}_{\mathbf{r}}, \bar{\xi}_{\mathbf{r}}{ }^{*}, \overline{\partial \alpha}_{\mathbf{r}}$, and $\overline{\partial \beta}_{\mathbf{r}}{ }^{*}$ have to be calculated self-consistently and are explicitly given in the Appendix A. Notice that the last line of Eq. 19 couples the time derivative of the coefficient $f_{\mathrm{n}}^{(\mathbf{r})}$ with the coefficients $f_{\mathrm{n} \pm 2}^{(\mathbf{r})}$, the coupling being proportional to $J^{2} / U$. In particular this is different than the standard mean-field equations where the time derivative of the coefficient $f_{\mathrm{n}}^{(\mathbf{r})}$ is at most coupled to the coefficients $f_{\mathrm{n} \pm 1}^{(\mathbf{r})}$ through $J$. It is worth noting that Eq. 19 conserve the total number of particles for any $J(t)$. In what follows, we shall obtain a numerical solution of Eq. 19] to address the dynamics of a translationally invariant Bose-Hubbard model both for sudden quench and non-linear ramp of $J(t)$.

\section{A. Sudden Quench}

In this section, we are going to address the dynamics of the bosons after a sudden quench of the hopping amplitude from $J_{i}$ (Mott phase) to $J_{f}$ (superfluid phase) through the tip of the Mott lobe where the dynamical critical exponent $z=1$. Our main objective here is to compute the time evolution of the order parameter $\Delta_{\mathbf{r}}(t)=\left\langle\psi(t)\left|b_{\mathbf{r}}\right| \psi(t)\right\rangle$ and the order-parameter correlation function $C_{\mathbf{r}}(t)=\left\langle\psi(t)\left|b_{\mathbf{r}} b_{\mathbf{r}}\right| \psi(t)\right\rangle-\Delta_{\mathbf{r}}^{2}(t)$. We shall also consider sudden quenches which start at the critical point $\left(J_{i}=J_{c}\right)$ and end in the superfluid phase $J_{f}>J_{c}$, and compute the resultant residual energy $Q$ and the wavefunction overlap $F$.

To this end, we begin by noting that for a sudden quench, $\partial J / \partial t \sim \delta(t)$ and thus the first term on the right side of Eq.19] does not contribute to the subsequent timeevolution of the system for $t>0$. The time evolution of the order parameter $\Delta(t)$ can then be written in terms of $\left\{f_{n}(t)\right\}$ by noting that $\Delta(t)=\left\langle\psi^{\prime}(t)\left|b_{\mathbf{r}}^{\prime}\right| \psi^{\prime}(t)\right\rangle$, where $b_{\mathbf{r}}^{\prime}=\exp \left(i S\left[J_{f}\right]\right) b_{\mathbf{r}} \exp \left(-i S\left[J_{f}\right]\right)$. One can then express $\Delta(t)$ in terms of $f_{n}^{(\mathbf{r})}$ as

$$
\begin{aligned}
\Delta_{\mathbf{r}}(t) & =\varphi_{\mathbf{r}}(t)+J / U \sum_{\left\langle\mathbf{r}^{\prime}\right\rangle_{\mathbf{r}}} \bar{n}\left[\left|f_{\bar{n}}^{(\mathbf{r})}\right|^{2}-\left.f_{\bar{n}-1}^{(\mathbf{r})}\right|^{2}\right] \varphi_{\mathbf{r}^{\prime} \bar{n}} \\
& +(\bar{n}+1)\left[\left|f_{\bar{n}}^{(\mathbf{r})}\right|^{2}-\left.f_{\bar{n}+1}^{(\mathbf{r})}\right|^{2}\right] \varphi_{\mathbf{r}^{\prime}, \bar{n}-1}+\left[\Phi_{\mathbf{r}, \bar{n}-2}\right. \\
& \left.-\Phi_{\mathbf{r}, \bar{n}-1}\right] \varphi_{\mathbf{r}^{\prime} \bar{n}}^{*}+\left[\Phi_{\mathbf{r} \bar{n}}-\Phi_{\mathbf{r}, \bar{n}-1}\right] \varphi_{\mathbf{r}^{\prime}, \bar{n}-1}^{*} \cdot
\end{aligned}
$$

Note that the first term in Eq. 20 represents the meanfield result while the presence of the other terms indicate contribution from the quantum fluctuations from meanfield theory. The role of such quantum fluctuations in the evolution of $\Delta_{\mathbf{r}}(t)$ becomes evident in computing the equal-time order parameter correlation function $C_{\mathbf{r}}(t)$. To compute $\Delta_{\mathbf{r}}$ and $C_{\mathbf{r}}$, we consider a spatially homogeneous system and solve the Schrödinger equation (Eq.19) for $f_{n}^{(\mathbf{r})} \equiv f_{n}$ (as guaranteed by translational invariance) keeping all states for $0 \leq n \leq 5$ with $\bar{n}=1$. The resultant plot of $\Delta_{\mathbf{r}}(t) \equiv \Delta(t)$ is shown in Fig. [5(a) [(d)] for $J_{i}=0$ and $J_{f} / J_{c}=1.02\left(J_{f} / J_{c}=3.51\right)$. We find that near the critical point, $\Delta(t)$ displays oscillations with a single characteristic frequency 21 while away from the critical point $\left(J_{f} / J_{c}=3.51\right)$, multiple frequencies are involved in its dynamics. The time period $T$ (Fig. 5 (c)) of these oscillations near $J_{c}$ is found, as a consequence of critical slowing down, to have a divergence $T \sim(\delta J)^{-0.35 \pm 0.05}$ leading to $z \nu=0.35 \pm 0.05$ for $d=3 \frac{13,28}{}$. Finally, we plot $C_{\mathbf{r}}(t) \equiv C(t)$ as a function of $t$ for $J_{f}=1.02 J_{c}$ in 
Fig. 5(b). We find that $\left|C(t) / \Delta^{2}(t)\right|$ may be as large as 0.5 at the tip of the peaks of $\Delta(t)$, which shows strong quantum fluctuations near the QCP.

To compare our results with the order parameter dynamics obtained from the mean-field theory, we solve the equations of motion for the time dependent Gutzwiller coefficients $f_{n}(t)$ within a single-site mean-field theory. As shown in Ref. 21,23, the mean-field equation reads

$$
\begin{aligned}
\left(i \partial_{t}-\epsilon_{n}\right) f_{n}= & -z_{0} J(t)\left[\Delta_{\mathrm{mf}}(t) \sqrt{n} f_{n-1}\right. \\
& \left.+\Delta_{\mathrm{mf}}^{*}(t) \sqrt{n+1} f_{n+1}\right]
\end{aligned}
$$

where $\epsilon_{n}=-\mu n+U n(n-1) / 2$ is the on-site energy of the bosons, $\Delta_{\mathrm{mf}}(t)=\sum_{n} f_{n-1}^{*} f_{n} \sqrt{n}$, and $J(t)=\left(J_{i} \theta(-t)+\right.$ $\left.J_{f} \theta(t)\right)$ for the sudden quench protocol. For the meanfield theory the critical point lies at $J_{c}^{\mathrm{mf}}=0.028 U$ for $d=3$. To obtain the order parameter dynamics, we obtain the values of $f_{n}(t)$ numerically keeping up to $n=5$ states and compute the order parameter $\Delta_{\mathrm{mf}}(t)$ choosing the same sudden quench protocol used in the projection operator approach (see Fig. (5)). The behavior of $\Delta_{\mathrm{mf}}(t)$ as a function of time is shown in the left panel Fig. 6. A comparison of our results with that of the mean-field theory can now be made by comparing Figs. 5 and 6 , We find that although the qualitative nature of $\Delta_{\mathrm{mf}}(t)$ and $\Delta(t)$ are similar, the periodicity of the oscillations are quite different. Further we note that

$$
\begin{aligned}
C_{\mathrm{mf}}(t) & =\left\langle b_{\mathbf{r}}^{2}\right\rangle-\Delta_{\mathrm{mf}}^{2}(t) \\
& =\sum_{n} \sqrt{n(n-1)} f_{n-2}^{*} f_{n}-\Delta_{\mathrm{mf}}^{2}(t)
\end{aligned}
$$

is also expected to show qualitatively similar behavior to $C(t)$. Thus we conclude that the subsequent dynamics of the order parameter following a quantum quench near a critical point is qualitatively similar in nature to what is found from mean-field theory; however, the precise quantitative value of, for example, its period of oscillation, receives significant contribution from quantum fluctuations.

Next, we compute the wavefunction overlap $F=$ $\left|\left\langle\psi_{f} \mid \psi_{c}\right\rangle\right|^{2}=\left|\left\langle\psi_{f}^{\prime}\left|e^{i S\left[J_{f}\right]} e^{-i S\left[J_{c}\right]}\right| \psi_{c}^{\prime}\right\rangle\right|^{2}$ for sudden quench starting at the QCP. Here $\psi_{f}\left(\psi_{c}\right)$ denotes the ground state wavefunction for $J=J_{f}\left(J_{c}\right)$. The residual energy $Q=\left\langle\psi_{c}\left|\mathcal{H}\left[J_{f}\right]\right| \psi_{c}\right\rangle-E_{G}\left[J_{f}\right]$, where $E_{G}\left[J_{f}\right]$ denotes the ground state energy at $J=J_{f}$ as obtained by minimizing $E$ in Eq. 9, can also be computed in a similar manner. Using the fact that for $\left|\psi_{c}^{\prime}\right\rangle=e^{i S\left[J_{c}\right]}\left|\psi_{c}\right\rangle, \varphi_{\mathbf{r}}=\Phi_{\mathbf{r}}=0$, we find, in terms of the coefficients $f_{n}^{(\mathbf{r})}$,

$$
\begin{aligned}
Q= & E_{G}\left[J_{c}\right]-E_{G}\left[J_{f}\right]-2 J \delta J \bar{n}(\bar{n}+1) \sum_{\left\langle\mathbf{r r}^{\prime}\right\rangle}\left[\left|f_{\bar{n}}^{(\mathbf{r})}\right|^{2}\left|f_{\bar{n}}^{\left(\mathbf{r}^{\prime}\right)}\right|^{2}\right. \\
& \left.-\left|f_{\bar{n}+1}^{(\mathbf{r})}\right|^{2}\left|f_{\bar{n}-1}^{\left(\mathbf{r}^{\prime}\right)}\right|^{2}-f_{\bar{n}+1}^{*(\mathbf{r})} f_{\bar{n}-1}^{(\mathbf{r})} f_{\bar{n}-1}^{*\left(\mathbf{r}^{\prime}\right)} f_{\bar{n}+1}^{\left(\mathbf{r}^{\prime}\right)}\right] / U .
\end{aligned}
$$

A plot of $1-F$ and $Q$ for the homogeneous case, as a function of $\delta J$ for $\delta J / J_{c} \lesssim 0.2$ is shown in Fig. 7. A
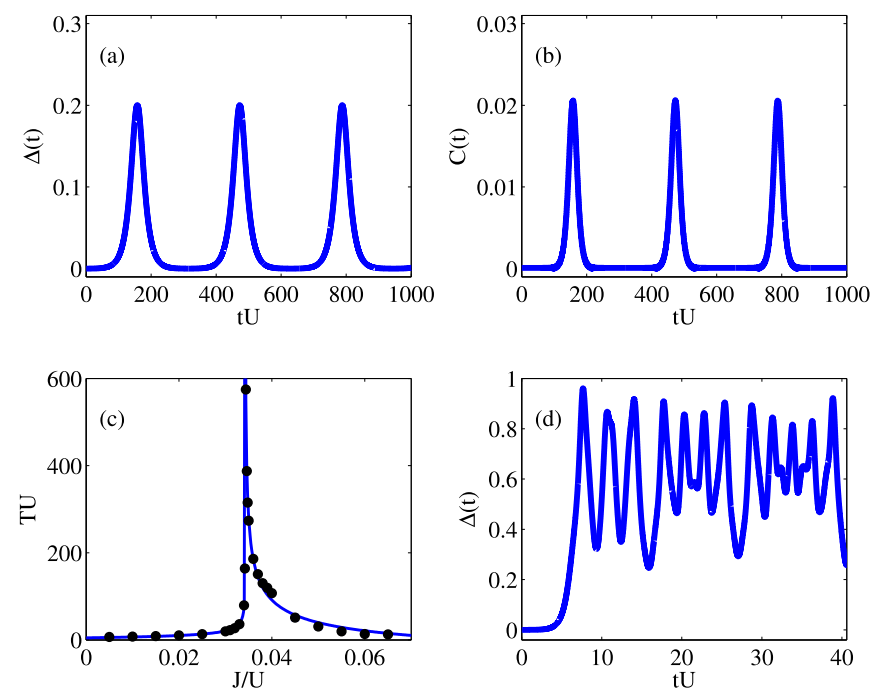

FIG. 5: (Color online) Plot of $\Delta(t)$ (a), and $C(t)(\mathrm{b})$ as a function of $t U$, for $J_{f}=1.02 J_{c}$. (c) The time period $T$ of the oscillations of $\Delta(t)$. (d) Same as in (a) for $J_{f}=3.51 J_{c}$. We have set $\hbar=1$ for all plots.
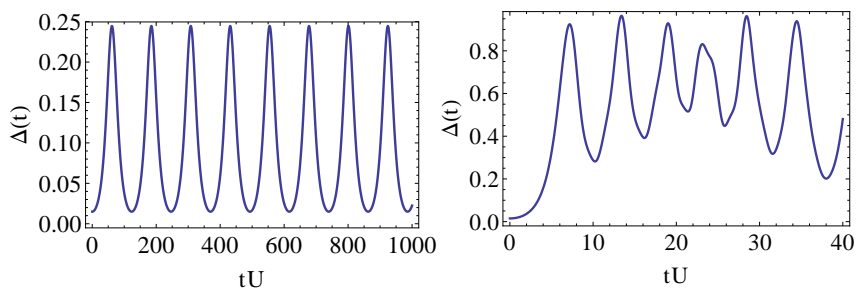

FIG. 6: (Color online) Plot of $\Delta(t)$ as a function of $U t(\hbar=1)$ as computed using mean-field theory for $J_{f}=1.02 J_{c}^{\mathrm{mf}}$ (left panel) and $J_{f}=3.51 J_{c}^{\mathrm{mf}}$ (right panel).

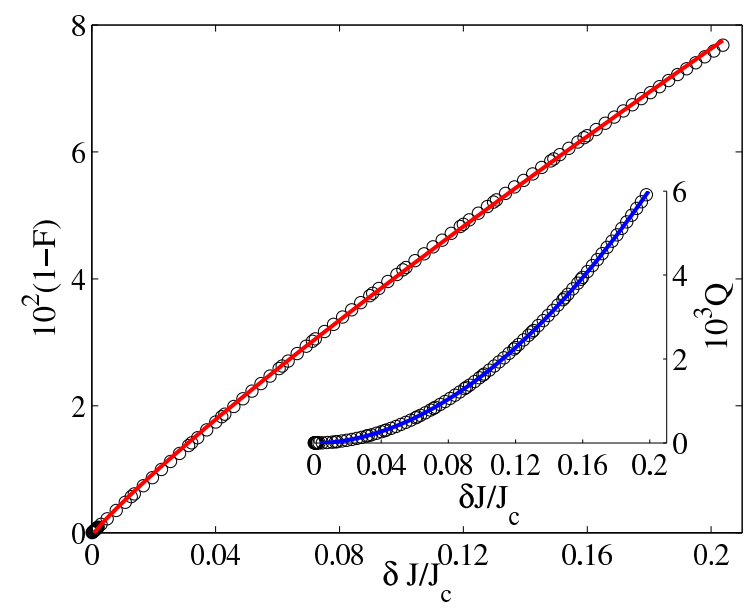

FIG. 7: (Color online) Plot of $F$ and $Q$ as a function of the $\delta J$ for $\delta J / J_{c} \ll 1$. The lines correspond to fits yielding a power $1-F(Q) \sim(\delta J)^{r_{1}\left(r_{2}\right)}$ with $r_{1} \simeq 0.89$ and $r_{2} \simeq 1.9$. 
numerical fit of these curves yields $1-F \sim \delta J^{0.89}$ and $Q \sim \delta J^{1.90}$ which disagrees with the universal scaling exponents $\left(1-F \sim \delta J^{d \nu}\right.$ and $\left.Q \sim \delta J^{(d+z) \nu}\right)$ expected from sudden dynamics across a QCP with $z=1^{29}$. In the next section, we shall study ramp dynamics across the quantum critical point and try to understand the reason behind such lack of universality in the dynamics of the bosons.

Before ending this section, we note that it is possi- ble to compute the evolution of the correlator $B_{\ell}=$ $\left\langle\left(b_{\mathbf{r}}^{\dagger} b_{\mathbf{r}^{\prime}}+\right.\right.$ h.c. $\left.)\right\rangle\left(\ell\right.$ is the link between sites $\mathbf{r}$ and $\left.\mathbf{r}^{\prime}\right)$ after a sudden quench from $J=J_{i}$ to $J_{f}$ where $J_{i}$ corresponds to the MI state and $J_{f}$ correspond to either the SF phase or the MI phase. The mean-field results for such a correlation would be zero if $J_{f}$ corresponds to the MI state and $\left|\Delta^{2}(t)\right|$ if it corresponds to a homogeneous SF phase. In contrast, using Eq. 10 with $J=J_{f}$, we find that the projection operator approach yields,

$$
\begin{aligned}
B_{\ell}= & \Re\left(\varphi_{\mathbf{r}}^{*} \varphi_{\mathbf{r}^{\prime}}\right)+\frac{J_{f}}{U}\left[\Re\left(\Phi_{\mathbf{r}, \bar{n}-2}^{*} \Phi_{\mathbf{r}^{\prime}, \bar{n}}+\Phi_{\mathbf{r}, \bar{n}} \Phi_{\mathbf{r}^{\prime}, \bar{n}-2}^{*}\right)+2 \bar{n}(\bar{n}+1)\left|f_{\bar{n}}^{(\mathbf{r})}\right|^{2}\left|f_{\bar{n}}^{\left(\mathbf{r}^{\prime}\right)}\right|^{2}\right. \\
& -\bar{n}(\bar{n}+1)\left(f_{\bar{n}-1}^{(\mathbf{r})} f_{\bar{n}+1}^{(\mathbf{r}) *} f_{\bar{n}+1}^{\left(\mathbf{r}^{\prime}\right)} f_{\bar{n}-1}^{\left(\mathbf{r}^{\prime}\right) *}+f_{\bar{n}+1}^{(\mathbf{r})} f_{\bar{n}-1}^{(\mathbf{r}) *} f_{\bar{n}-1}^{\left(\mathbf{r}^{\prime}\right)} f_{\bar{n}+1}^{\left(\mathbf{r}^{\prime}\right) *}+\left|f_{\bar{n}+1}^{(\mathbf{r})}\right|^{2}\left|f_{\bar{n}-1}^{\left(\mathbf{r}^{\prime}\right)}\right|^{2}\right. \\
& \left.\left.+\left|f_{\bar{n}-1}^{(\mathbf{r})}\right|^{2}\left|f_{\bar{n}+1}^{\left(\mathbf{r}^{\prime}\right)}\right|^{2}\right)\right]+\frac{J_{f}}{U} \sum_{\left\langle\mathbf{r r}^{\prime} \mathbf{r}^{\prime \prime}\right\rangle}\left\{2 \Re \left[\varphi_{\mathbf{r}, \bar{n}-1}^{*}(\bar{n}+1)\left|f_{\bar{n}}^{\left(\mathbf{r}^{\prime}\right)}\right|^{2}\right.\right. \\
& \left.+\varphi_{\mathbf{r} \bar{n}} \Phi_{\mathbf{r}^{\prime}, \bar{n}-2}^{*}-\varphi_{\mathbf{r} \bar{n}}^{*} \bar{n}\left|f_{\bar{n}-1}^{\left(\mathbf{r}^{\prime}\right)}\right|^{2}-\varphi_{\mathbf{r}, \bar{n}-1} \Phi_{\mathbf{r}^{\prime}, \bar{n}-1}^{*}\right] \varphi_{\mathbf{r}^{\prime \prime}}+2 \Re\left[\varphi_{\mathbf{r}, \bar{n}-1}^{*} \Phi_{\mathbf{r}^{\prime} \bar{n}}+\varphi_{\mathbf{r} \bar{n}} \bar{n}\left|f_{\bar{n}}^{\left(\mathbf{r}^{\prime}\right)}\right|^{2}\right. \\
& \left.\left.-\varphi_{\mathbf{r}_{\bar{n}}}^{*} \Phi_{\mathbf{r}^{\prime}, \bar{n}-1}-\varphi_{\mathbf{r}, \bar{n}-1}(\bar{n}+1)\left|f_{\bar{n}+1}^{\left(\mathbf{r}^{\prime}\right)}\right|^{2}\right] \varphi_{\mathbf{r}^{\prime \prime}}^{*}\right\}
\end{aligned}
$$
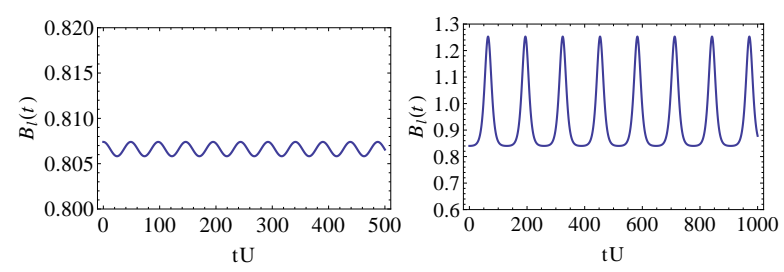

FIG. 8: (Color online) Plot of $B_{\ell}(t)$ after a sudden quench from $J_{i}$ to $J_{f}$ as a function of time for $J_{f}=0.98 J_{c}$ (left panel) and $J_{f}=1.02 J_{c}$ (right panel). See text for details.

When $J_{f}$ correspond to the MI phase, since $f_{\bar{n}}(t) \gg$ $f_{n \neq \bar{n}}(t)$, we find that $B_{\ell}$ shows very small oscillations around the base value $4 J_{f}\left|f_{\bar{n}}\right|^{4} / U$. In contrast, it displays significant oscillation in the SF phase. These behaviors, in the homogeneous limit, are sketched in the left and right panels of Fig. 8 for $J_{f}=0.98 J_{c}$ (left panel) and $1.02 J_{c}$ (right panel). We note that the behavior of $B_{\ell}$ in the MI phase is qualitatively different from the mean-field result which correspond to the first term in the right side of Eq. 23.

\section{B. Non-linear Ramp}

In this section, we address the dynamics of the bosons during a ramp of the hopping amplitude $J$ characterized by a rate $\tau^{-1}$ and an exponent $\alpha: J(t)=J_{i}+\left(J_{f}-\right.$ $\left.J_{i}\right)(t / \tau)^{\alpha}$. Note that the system evolves from $J_{i}$ at $t_{i}=0$ to $J_{f}$ at $t_{f}=\tau$; consequently as long as we restrict our-

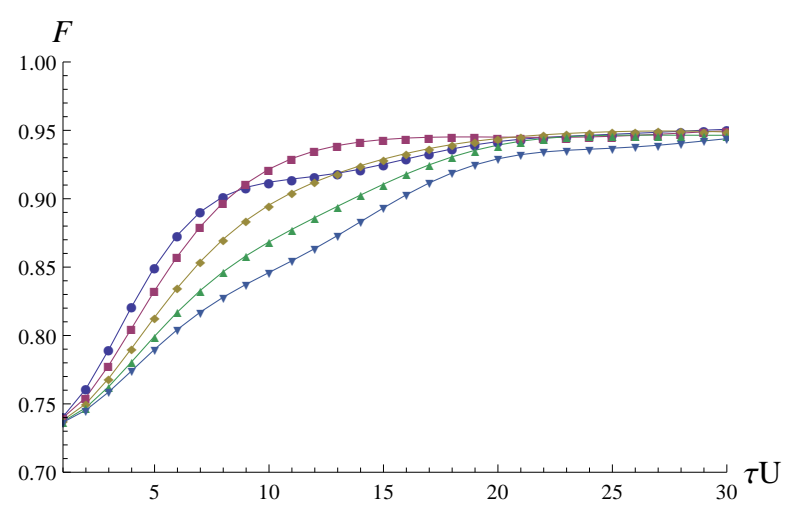

FIG. 9: (Color online) Plot of $F=1-P$ as a function $\tau U$ (in units of $\hbar=1$ ) for $J_{i} / U=0.05$ (SF phase) and $J_{f} / U=$ 0.005 (Mott phase) for $\alpha=1$ (blue circles), 2 (red squares), 3 (yellow diamonds), 4 (green triangles), and 5 (blue inverted triangles) showing the plateau-like behavior at large $\tau$.

selves to $J_{i} / U, J_{f} / U \ll 1$, we expect the perturbative projection method to address the dynamics accurately irrespective of the values of $\tau$ and $\alpha$. Thus the projection operator method enables one to address "slow" and "fast" and linear/non-linear ramps at equal footing. We note at the outset that our results in this section reproduce those in Ref. 24 as a special case for $\alpha=1$.

To address the dynamics, we use Eq. 19 and solve for $f_{n}^{(\mathbf{r})} \equiv f_{n}$ for translationally invariant systems. This enables us to compute the defect formation probability $P=1-\left|\left\langle\psi_{G} \mid \psi\left(t_{f}\right)\right\rangle\right|^{2}=1-F$, where $\left|\psi_{G}\right\rangle\left(\left|\psi\left(t_{f}\right)\right\rangle\right)$ 


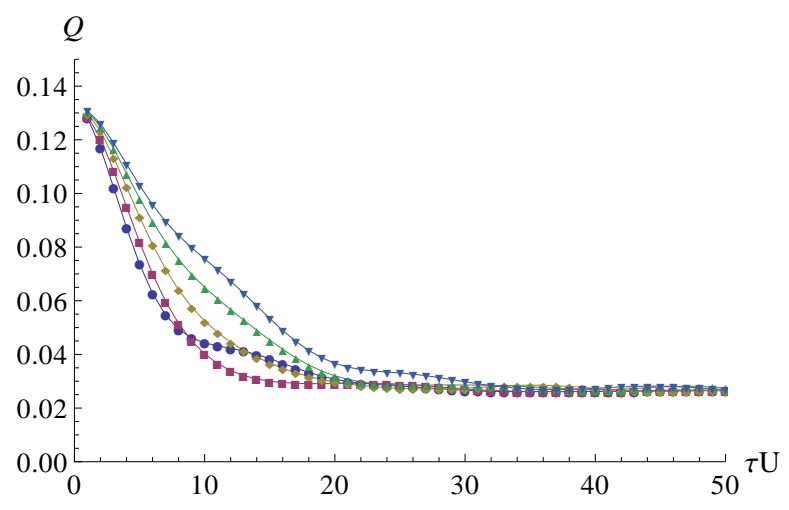

FIG. 10: (Color online) Plot of $Q$ as a function of $\tau$ for $\alpha=$ 1..5. All parameters and symbols are same as those in Fig. 9

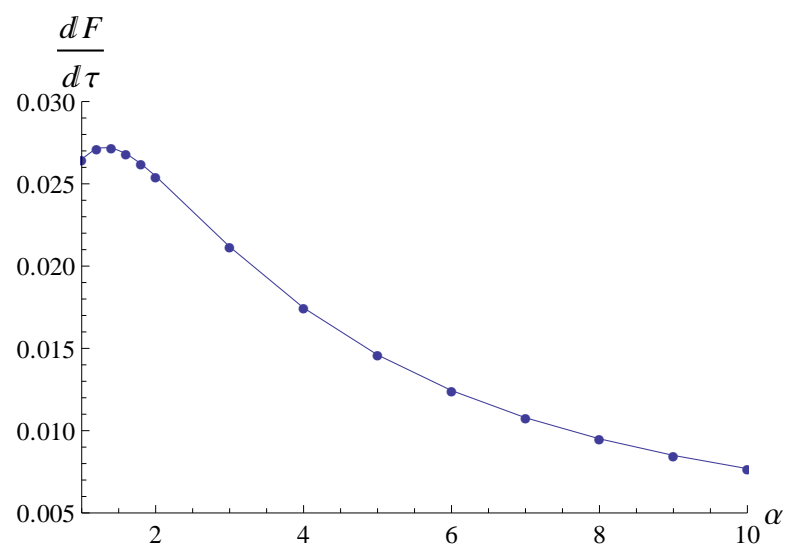

FIG. 11: (Color online) Plot of $d F / d \tau$ for small $\tau(\tau U \leq 5)$ as a function of $\alpha$.

denotes the final ground state (state after the ramp), for a ramp from $J_{i} / U=0.05$ (superfluid phase) to $J_{f} / U=0.005$ (Mott phase) as a function of $\tau$. The behavior of $F$ and $Q$ are shown in Figs. 9 and 10 for various representative values of $\alpha$. We find that both $Q$ and $F$ (and hence $P$ ) exhibits a plateau like behavior at large $\tau$. The slope of both $F$ and $Q$ for small $\tau$ depend on the ramp protocol through the exponent $\alpha$; however, the asymptotic values of these quantities at large $\tau$ is independent of $\alpha$. The plot of $d F / d \tau$ for $\tau U \leq 5$ (where $F$ is approximately linear in $\tau$ as can be seen from Fig. 9) is shown in Fig. 11] The slope decreases monotonically with $\alpha$ for large $\alpha$ which indicates that $F$ (and similarly $Q$ ) saturates at larger values of $\tau$ with increasing $\alpha$. The slope is maximal, indicating minimal initial defect production, for $\alpha=1.5$.

It is clear from the plots that both $P$ and $Q$ do not display universal scaling as expected from generic theories of slow dynamics of quantum systems near critical point ${ }^{13}$. This seems to be in qualitative agreement with the recent experiments presented in Ref. 2, where linear ramp dynamics of ultracold bosons from superfluid to the Mott region has been experimentally studied. Indeed, it was found, via direct measurement of parity of $\bar{n}$ per site, that $F$ displays a plateau like behavior similar to Fig. 9 , Such a lack of universality in the dynamics can be qualitatively understood from absence of contribution of the critical $(\mathbf{k}=0)$ modes. In the strong-coupling regime $\left(z_{0} J / U \ll 1\right)$, the system can access the $\mathbf{k}=0$ modes after time $\mathcal{T}$ which can be roughly estimated as the time taken by a boson to cover the linear system dimension $L$. For typical small $J(U=1)$ in the Mott phase and near the QCP, $\mathcal{T} \sim \mathrm{O}(L \hbar / J)$ can be very large. Thus for $t \leq \mathcal{T}$, the dynamics, governed by local physics which is well captured by our method, do not display critical scaling behavior. We note that our theory which is based on building on spatial correlation order by order in powers of $z_{0} J / U$ shall not easily capture the physics associated with long-range spatial correlation near the critical point and will deviate from experimental results for much slower ramp rates. It seems, however, that achieving such low ramp rates for the present system in the Mott phase can be experimentally challenging.

\section{DISCUSSION}

In conclusion, we have presented a projection operator formalism that describes in a semi-analytical way both the phase diagram and non-equilibrium dynamics of the Bose-Hubbard model. It produces a phase diagram which is nearly identical to the QMC results in $3 \mathrm{D}$, allows for a computation of the low-energy excitation spectra of the system, and yields semi-analytical insight for several quantities such as $F, Q, \Delta(t), P$, and $C(t)$ for non-equilibrium dynamics. Its prediction for $P$ for a slow ramp matches qualitatively with recent experiments. The method, in principle, can be generalized to any strongly correlated systems which allows perturbative treatment of fluctuations. We leave such considerations for future study. We also note that studying finite temperature physics of the Bosons with our method also poses an interesting theoretical challenge. For now, we can only estimate the range of physical temperatures $T$ for which the $T=0$ theory is accurate. For a typical lattice depth in the Mott or critical regime, one can estimate $U \sim 2 \mathrm{kHz} \simeq 200 \mathrm{nK}^{1}$. This yields, in $3 \mathrm{D}$, a melting temperature $T^{*} \simeq 0.2 U=40 \mathrm{nK}$ for the Mott phase and critical temperature $T_{c} \simeq z_{0} J_{c} \simeq 35 \mathrm{nK}$ for the SF phase at Mott tip $\underline{30}$. This necessitates $T \ll T_{c}, T^{*}$ to be a few nano-Kelvins which is well within the current experimental limit $\sim 1 \mathrm{nK}^{30}$.

CT thanks M. Lewenstein for support during the work. The authors thank E. Altman, C. Lannert, S. Mondal, A. Polkovnikov, R. Sensarma, and S. Vishveshwara for discussions on related topics, and B. Caprogrosso-Sansone for sharing QMC data. KS thanks DST, India for support under Project No. SR/S2/CMP-001/2009. CT acknowledges support of Spanish MEC (FIS2008-00784, QOIT) and hospitality of Theoretical Physics Department, IACS. 


\section{Appendix A: Explicit form of the fields}

In this section we provide explicit expressions for the fields used in Eq 19. In what follows we define $\delta_{i j}$ to be the Kronecker delta and $\Lambda_{i j}=1-\delta_{i j}$. The fields that multiply the time derivative of the tunneling coefficient are given by

$$
\begin{aligned}
\overline{\partial \alpha}_{i} & =\sum_{\langle b\rangle_{i}}\left(\delta_{n \bar{n}} \varphi_{b \bar{n}}-\delta_{n, \bar{n}+1} \varphi_{b, \bar{n}-1}\right), \\
\overline{\partial \beta}_{i}^{*} & =\sum_{\langle b\rangle_{i}}\left(\delta_{n \bar{n}} \varphi_{b, \bar{n}-1}^{*}-\delta_{n, \bar{n}-1} \varphi_{b \bar{n}}^{*}\right) .
\end{aligned}
$$

The phase factor $\chi_{\mathrm{n}}^{(i)}$ is given by

$$
\begin{gathered}
\chi_{\mathrm{n}}^{(i)}=-\mu n+\frac{U}{2} n(n-1)-\frac{2 J^{2}}{U} \delta_{n \bar{n}} \bar{n}(\bar{n}+1) \sum_{\langle a\rangle_{i}}\left|f_{\bar{n}}^{(a)}\right|^{2} \\
-\frac{2 J^{2}}{U} \sum_{\langle a\rangle_{i}} \sum_{\langle c\rangle_{i}} \Lambda_{a c}\left[\bar{n}\left(\delta_{n \bar{n}}-\delta_{n, \bar{n}-1}\right) \Re \varphi_{a \bar{n}}\left(\varphi_{c}^{*}-\frac{\varphi_{c \bar{n}}^{*}}{2}\right)\right. \\
\left.+(\bar{n}+1)\left(\delta_{n \bar{n}}-\delta_{n, \bar{n}+1}\right) \Re \varphi_{a, \bar{n}-1}\left(\varphi_{c}^{*}-\frac{\varphi_{c, \bar{n}-1}^{*}}{2}\right)\right] \\
+\frac{J^{2}}{U} \bar{n}(\bar{n}+1)\left[\delta_{n, \bar{n}+1} \sum_{\langle a\rangle_{i}}\left|f_{\bar{n}-1}^{(a)}\right|^{2}+\delta_{n, \bar{n}-1} \sum_{\langle a\rangle_{i}}\left|f_{\bar{n}+1}^{(a)}\right|^{2}\right] .
\end{gathered}
$$

Notice that $\chi_{\mathrm{n}}^{(i)}$ gives only a phase factor in real time dynamics and is therefore negligible; however, it is important in the imaginary time evolution. The fields $\bar{\alpha}_{\mathbf{r}}$ and $\bar{\beta}_{\mathbf{r}}{ }^{*}$, which couples $\partial_{t} f_{\mathrm{n}}^{(\mathbf{r})}$ to $f_{\mathrm{n} \pm 1}^{(\mathbf{r})}$ linearly in $J$ are given by:

$$
\begin{array}{r}
\bar{\alpha}_{i}=\sum_{\langle a\rangle_{i}}\left[\varphi_{a}+\frac{J}{U} \mathcal{A}_{a}^{(i)}-\delta_{n \bar{n}}\left(\varphi_{a \bar{n}}-\frac{J}{U} \mathcal{B}_{a}^{(i)}\right)\right. \\
\left.-\delta_{n, \bar{n}+1}\left(\varphi_{a, \bar{n}-1}-\frac{J}{U} \mathcal{C}_{a}^{(i)}\right)\right],
\end{array}
$$

and

$$
\begin{array}{r}
\bar{\beta}_{i}{ }^{*}=\sum_{\langle a\rangle_{i}}\left[\varphi_{a}^{*}+\frac{J}{U} \mathcal{A}_{a}^{*(i)}-\delta_{n, \bar{n}-1}\left(\varphi_{a \bar{n}}^{*}-\frac{J}{U} \mathcal{B}_{a}^{*(i)}\right)\right. \\
\left.-\delta_{n \bar{n}}\left(\varphi_{a, \bar{n}-1}^{*}-\frac{J}{U} \mathcal{C}_{a}^{*(i)}\right)\right] .
\end{array}
$$

In contrast, the fields $\bar{\eta}_{\mathbf{r}}$ and $\bar{\xi}_{\mathbf{r}}^{*}$, which couple $\partial_{t} f_{\mathrm{n}}^{(\mathbf{r})}$ to $f_{\mathrm{n} \pm 2}^{(\mathbf{r})}$ to $\mathrm{O}\left(J^{2} / U\right)$ are given by

$$
\begin{gathered}
\bar{\eta}_{i}=\sum_{\langle a\rangle_{i}}\left\{\delta_{n \bar{n}} \Phi_{a \bar{n}}+\delta_{n, \bar{n}+2} \Phi_{a, \bar{n}-2}-\delta_{n, \bar{n}+1} \Phi_{a, \bar{n}-1}\right. \\
+\sum_{\langle c\rangle_{i}} \Lambda_{a c}\left(\delta_{n \bar{n}} \varphi_{a \bar{n}}+\delta_{n, \bar{n}+2} \varphi_{a, \bar{n}-1}\right) \varphi_{c} \\
\left.-\delta_{n, \bar{n}+1} \sum_{\langle c\rangle_{i}} \Lambda_{a c}\left[\left(\varphi_{a \bar{n}}+\varphi_{a, \bar{n}-1}\right) \varphi_{c}-\varphi_{a \bar{n}} \varphi_{c, \bar{n}-1}\right]\right\},
\end{gathered}
$$

and

$$
\begin{gathered}
\bar{\xi}_{i}^{*}=\sum_{\langle a\rangle_{i}}\left\{\delta_{n, \bar{n}-2} \Phi_{a \bar{n}}^{*}+\delta_{n \bar{n}} \Phi_{a, \bar{n}-2}^{*}-\delta_{n, \bar{n}-1} \Phi_{a, \bar{n}-1}^{*}\right. \\
+\sum_{\langle c\rangle_{i}} \Lambda_{a c}\left(\delta_{n, \bar{n}-2} \varphi_{a \bar{n}}^{*}+\delta_{n \bar{n}} \varphi_{a, \bar{n}-1}^{*}\right) \varphi_{c}^{*} \\
\left.-\delta_{n, \bar{n}-1} \sum_{\langle c\rangle_{i}} \Lambda_{a c}\left[\left(\varphi_{a \bar{n}}^{*}+\varphi_{a, \bar{n}-1}^{*}\right) \varphi_{c}^{*}-\varphi_{a \bar{n}}^{*} \varphi_{c, \bar{n}-1}^{*}\right]\right\},
\end{gathered}
$$

where we have introduced the quantities

$$
\begin{gathered}
\mathcal{A}_{a}^{(i)}=\sum_{\langle b\rangle_{a}} \Lambda_{b i}\left[\left(\Phi_{a, \bar{n}-2}-\Phi_{a, \bar{n}-1}\right) \varphi_{b \bar{n}}^{*}+\left(\Phi_{a \bar{n}}-\Phi_{a, \bar{n}-1}\right) \varphi_{b, \bar{n}-1}^{*}\right. \\
\left.+(\bar{n}+1)\left(\left|f_{\overline{\mathrm{n}}}^{(a)}\right|^{2}-\left|f_{\overline{\mathrm{n}}+1}^{(a)}\right|^{2}\right) \varphi_{b, \bar{n}-1}+\bar{n}\left(\left|f_{\overline{\mathrm{n}}}^{(a)}\right|^{2}-\left|f_{\overline{\mathrm{n}}-1}^{(a)}\right|^{2}\right) \varphi_{b \bar{n}}\right] \\
\mathcal{B}_{a}^{(i)}= \\
\sum_{\langle b\rangle_{a}} \Lambda_{b i}\left[(\bar{n}+1)\left(\left|f_{\overline{\mathrm{n}}}^{(a)}\right|^{2}-\left|f_{\overline{\mathrm{n}}+1}^{(a)}\right|^{2}\right)\left(\varphi_{b}-\varphi_{b, \bar{n}-1}\right)\right. \\
\left.+\left(\Phi_{a \bar{n}}-\Phi_{a, \bar{n}-1}\right) \varphi_{b}^{*}+\Phi_{a, \bar{n}-1} \varphi_{b \bar{n}}^{*}\right],
\end{gathered}
$$

and

$$
\begin{aligned}
\mathcal{C}_{a}^{(i)}= & \sum_{\langle b\rangle_{a}} \Lambda_{b i}\left[\bar{n}\left(\left|f_{\overline{\mathrm{n}}}^{(a)}\right|^{2}-\left|f_{\overline{\mathrm{n}}-1}^{(a)}\right|^{2}\right)\left(\varphi_{b}-\varphi_{b \bar{n}}\right)\right. \\
& \left.+\left(\Phi_{a, \bar{n}-2}-\Phi_{a, \bar{n}-1}\right) \varphi_{b}^{*}+\Phi_{a, \bar{n}-1} \varphi_{b, \bar{n}-1}^{*}\right],
\end{aligned}
$$

for notational convenience.
1 M. Greiner, O. Mandel, T. Esslinger, T. W. Hnsch1 and I. Bloch Nature 415, 39 (2002);C. Orzel,A. K. Tuchman,M. L. Fenselau,M. Yasuda and M. A. Kasevich, Science 291, 2386 (2001); T. Kinoshita, T. Wenger, and D. S. Weiss, Nature 440, 900 (2006);L. E. Sadler, J. M. Higbie, S. R. Leslie, M. Vengalattore and D. M. Stamper-Kurn, Nature 443, 312 (2006).
2 W. S. Bakr, A. Peng, M. E. Tai, R. Ma, J. Simon, J. I. Gillen, S. Folling, L. Pollet, and M. Greiner, Science 329, 547 (2010).

${ }^{3}$ D. Jaksch, C. Bruder, J. I. Cirac, C. W. Gardiner, and P. Zoller, Phys. Rev. Lett. 81, 3108 (1998).

${ }^{4}$ M. P. A. Fisher, P. B. Weichman, G. Grinstein, and D. S. Fisher, Phys. Rev. B 40, 546 (1989). 
${ }^{5}$ K. Sheshadri, H. R. Krishnamurthy, R. Pandit, and T. V. Ramakrishnan, Europhys. Lett. 22, 257 (1993).

6 J. K. Freericks and H. Monien, Europhys. Lett. 26, 545 (1994); ibid, Phys. Rev. B 53, 2691 (1996).

7 W. Krauth and N. Trivedi, Europhys. Lett. 14, 627 (1991)

${ }^{8}$ K. Sengupta and N. Dupuis, Phys. Rev. A 71, 033629(2005).

9 A. Rancon and N. Dupuis, Phys. Rev. B 83, 172501 (2011); ibid., Phys. Rev. B 84, 174513 (2011).

10 See for example, M. Lewenstein, A. Sanpera, V. Ahufinger, B. Damskic, A. Sen(De) U. Sen Adv. Phys. 56,243 (2007).

11 B. Caprogrosso-Sansone, N. Prokofev, and B. V. Svistunov, Phys. Rev. B 75, 134302 (2007).

12 J. K. Freericks, H. R. Krishnamurthy, Y. Kato, N. Kawashima, and N. Trivedi, Phys. Rev. A 79, 053631 (2009).

13 A. Polkovnikov, K. Sengupta, A. Silva, and M. Vengalattore, Rev. Mod. Phys. 83, 863 (2011); J. Dziarmaga, Adv. Phys. 59, 1063 (2010).

14 A. Polkovnikov, Phys. Rev. B 72, 161201(R) (2005).

${ }^{15}$ K. Sengupta, D. Sen, and S. Mondal, Phys. Rev. Lett. 100, 077204 (2008); S. Mondal, D. Sen, and K. Sengupta, Phys. Rev. B 78, 045101 (2008).

16 D. Sen, K. Sengupta, and S. Mondal, Phys. Rev. Lett. 101, 016806 (2008); S. Mondal, K. Sengupta, and D. Sen, Phys. Rev. B 79, 045128 (2009);

17 F. Pellegrini, S. Montangero, G. E. Santoro, and R. Fazio, Phys. Rev. B 77, 140404(R) (2008); U. Divakaran, A. Dutta, and D. Sen, ibid. 78, 144301 (2008); U. Divakaran, V. Mukherjee, A. Dutta, and D. Sen, J. Stat. Mech. (2009) P02007.

18 C. Kollath, A. Lauchli, and E. Altman, Phys. Rev. Lett. 98, 180601 (2007).

${ }^{19}$ C. De Grandi, V. Gritsev, A. Polkovnikov, Phys. Rev. B
81, 224301 (2010); C. De Grandi, R. A. Barankov, and A. Polkovnikov, Phys. Rev. Lett. 101, 230402 (2008).

20 A. Polkovnikov, Phys. Rev. A 66, 053607 (2002); A. Polkovnikov and V. Gritsev, Nat. Phys. 4, 477 (2006).

21 E. Altman and A. Auerbach, Phys. Rev. Lett. 89, 250404 (2002).

22 M. P. Kennett and D. Dalidovich, Phys. Rev. A 84, 033620 (2011).

23 R. Schtzhold, M. Uhlmann, Y. Xu, and U. R. Fischer, Phys. Rev. Lett. 97, 200601 (2006); J. Wernsdorfer, M. Snoek, and W. Hofstetter Phys. Rev. A 81, 043620 (2010).

24 C. Trefzger and K. Sengupta, Phys. Rev. Lett. 106, 095702 (2011).

25 A. Isacsson, Min-Chul Cha, K. Sengupta, and S. M. Girvin, Phys. Rev. B 72, 184507 (2005).

26 C. Trefzger, C. Menotti, and M. Lewenstein, Phys. Rev. A 78, 043604 (2008).

27 K. V. Kruititsky and P. K. Navez, Phys. Rev. A 84, 033602 (2011).

28 Note that starting from perfect Mott state at $J=0$ where $f_{1}=1$ and $f_{n \neq 1}=0$ does not lead to any dynamics since all fields in the right side of Eq. 19 except $\chi_{n}(\mathbf{r})$ vanishes leading to a trivial phase-only contribution to $f_{1}$. This property can be seen to be a consequence of conservation of $n$ at all sites for any $J(t)$.

29 C. De Grandi, V. Gritsev, and A. Polkovnikov, Phys. Rev. B 81, 012303 (2010); C. de Grandi and A. Polkovnikov, Quantum Quenching, Annealing and Computation, Eds. A. Das, A. Chandra and B. K. Chakrabarti, Lect. Notes in Phys., 802 (Springer, Heidelberg 2010).

30 F. Gerbier, Phys. Rev. Lett. 99, 120405 (2007); D. M. Weld, P. Medley, H. Miyake, D. Hucul, D. E. Pritchard, and W. Ketterle, Phys. Rev. Lett. 103, 245301 (2009). 